\title{
Ferroelectric phase transitions induced by a strain gradient
}

\author{
P. Yudin $\odot,{ }^{1,2, *}$ J. Duchon,,${ }^{1}$ O. Pacherova $\odot,{ }^{1}$ M. Klementova,,${ }^{1}$ T. Kocourek,${ }^{1}$ A. Dejneka, ${ }^{1}$ and M. Tyunina $\odot{ }^{1,3, \dagger}$ \\ ${ }^{1}$ Institute of Physics, Academy of Sciences of the Czech Republic, Na Slovance 2, 18221 Praha 8, Czech Republic \\ ${ }^{2}$ Kutateladze Institute of Thermophysics, Siberian Branch of Russian Academy of Science, Lavrent'eva Ave. 1, Novosibirsk 630090, Russia \\ ${ }^{3}$ Microelectronics Research Unit, University of Oulu, P.O. Box 4500, FI-90014 Oulu, Finland
}

(Received 9 December 2020; revised 28 July 2021; accepted 30 July 2021; published 3 September 2021)

\begin{abstract}
In perovskite oxide ferroelectrics, gradients of lattice strain are known to induce nanoscale topological structures, leading to novel or enhanced functionality. Here, we experimentally detect and theoretically analyze thickness distribution of structural properties in epitaxial $\mathrm{Pb}_{0.5} \mathrm{Sr}_{0.5} \mathrm{TiO}_{3}$ films grown on (001) $\mathrm{SrTiO}_{3}$ substrates. We show that the relaxation of substrate-imposed stress produces a strain gradient, which leads to the formation of distinct ferroelectric phases as a function of distance from the film-substrate interface within the same film. Charge carriers trapped at phase boundaries stabilize the induced phases and manifest themselves under electric field. Crosstalk between the phases, where polarization may rotate in one phase and invert in the other one, opens perspectives for advanced ferroelectric thin film devices.
\end{abstract}

DOI: 10.1103/PhysRevResearch.3.033213

\section{INTRODUCTION}

Recent demand for nanotechnology has made nanoscale material science and physics critical areas of research. However, with our limited intuition for the nanoworld, understanding the structure-property relations often lags behind technological advances. While well-developed theories exist for bulk materials, extrapolation of these theories to thin films and nanostructures is frequently ambiguous. Thin films of perovskite oxide ferroelectrics are good examples of this, where advances in technology and research tools have opened horizons for experimental explorations, enabling progress in modeling and understanding [1-3].

Perovskite oxide ferroelectrics are widely studied for their diverse functionalities, including switchable polarization, piezoelectricity, high-dielectric susceptibility, pyroelectricity, electro-optics, and other effects that are essential for practical applications. The strong correlation between crystal structure and response functions is characteristic of these materials [4-7]. Therefore, an important option to control ferroelectric properties is by using lattice strain in epitaxial films [8]. Strain can efficiently enhance or tune major functional properties that are key for applications in actuation or sensing $[9,10]$, optics [11], and memory [12], to name a few.

Strain is typically imposed by selecting appropriate underlying single-crystal substrates. This approach is like that used in the semiconductor industry [13], called strain engineering

\footnotetext{
*yudin@fzu.cz

${ }^{\dagger}$ marina.tjunina@oulu.fi
}

Published by the American Physical Society under the terms of the Creative Commons Attribution 4.0 International license. Further distribution of this work must maintain attribution to the author(s) and the published article's title, journal citation, and DOI.
$[14,15]$. The magnitude of the achievable strain grows with the inverse of the film thickness and may exceed a few percent for the thinnest films.

In films thicker than a critical thickness of a few to a few tens of nanometers, a complex structure with lattice defects and inhomogeneous strain develops, typically resulting from dislocation-assisted strain relaxation but also with other multiple strain relaxation mechanisms, specific to growth conditions and material properties [16-18]. The critical thickness for these processes mainly depends on the film-substrate misfit strain and deposition temperature [19]. Whereas such defects as misfit and threading dislocations deteriorate the dielectric, piezoelectric, and ferroelectric properties of perovskite oxide thin films [20-23], the knowledge of defect physics may turn their presence into advantage. For instance, proper dislocation intensity can reduce the coercive field and enhance remnant polarization [24]; the strain gradient due to misfit dislocations can also enhance photonic behavior in $\mathrm{BiFeO}_{3}$ [25]. The coupling between the strain gradient and the electric polarization, or flexoelectricity, may yield even more advances and is an important growing field of study $[26,27]$. Strain gradients were also found to play a key role in the formation of nanoscale topology in ferroelectrics [28-31].

Our motivation for this paper was to discover nontrivial effects of strain and strain-gradient on structure and functionality of perovskite oxide films. In this respect, $\mathrm{Pb}_{0.5} \mathrm{Sr}_{0.5} \mathrm{TiO}_{3}$ (PSTO) appeared very promising, as its bulk samples exhibit a compositional paraelectric-to-ferroelectric transition at around $\mathrm{Sr} / \mathrm{Ti}=50 / 50$ [32]. These and other phases may simultaneously manifest themselves in an inhomogeneously strained film. We detected and analyzed phase changes that occur within epitaxial ferroelectric PSTO films grown on compressive $\mathrm{SrTiO}_{3}$ (STO) substrates. Crystal phases and strains as well as their distributions across the thickness of the film were established using x-ray and electron diffraction techniques and simulated using a phase field model. The fer- 

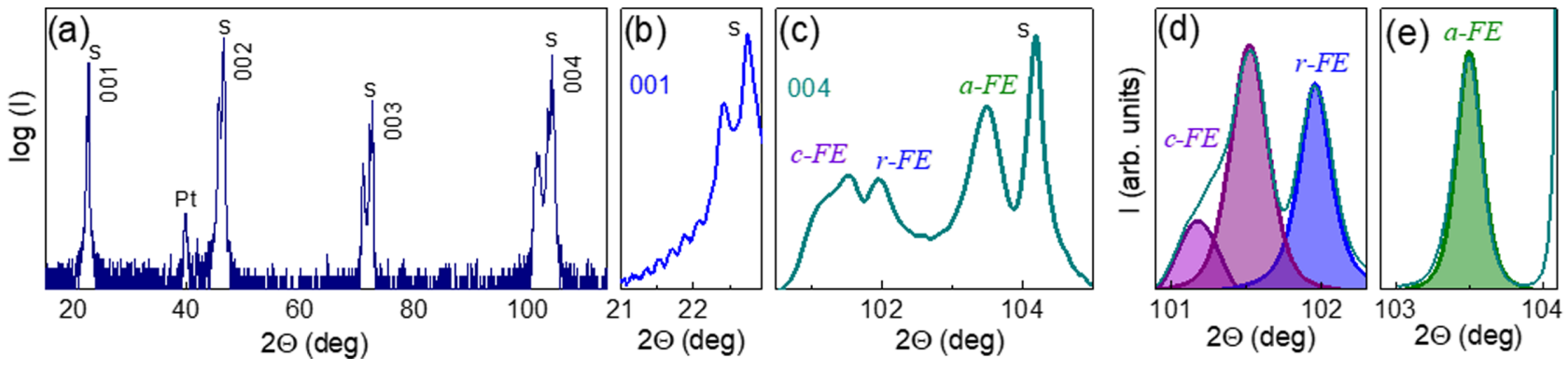

FIG. 1. X-ray diffraction (XRD) $\theta-2 \theta$ scans in (a) full range of $2 \theta$, (b) around (001) peak, and (c)-(e) around (004) peak. Peaks from substrate are marked by $s$ in (a)-(c). Peak fitting is shown in (d) and (e).

roelectric nature of the phases present in the film is proven by measurement of polarization-electric-field response. The interpretation of our results involves trapping of charge carriers at the interphase boundaries, which is of interest for applications in electronics. Relations of the results to flexoelectricity are discussed.

\section{METHODS}

\section{A. Experiment}

Thin films of ferroelectric PSTO and underlying conducting $\mathrm{SrRuO}_{3}$ (SRO) were grown by pulsed laser deposition using an excimer $\mathrm{KrF}$ laser (Compex $205 \mathrm{~F}$, wavelength $248 \mathrm{~nm}$, energy density $2 \mathrm{~J} / \mathrm{cm}^{2}$, pulse repetition rate $5 \mathrm{~Hz}$ ) on as-received $10 \times 10 \mathrm{~mm}^{2}$ single-crystal epitaxially polished (001) STO substrates (MTI Corp.). Dense ceramic pellets of PSTO and SRO synthesized in the Institute of Solid State Physics at the University of Latvia were used as targets. A substrate temperature of $973 \mathrm{~K}$ was used during deposition and lowered at a rate of $5 \mathrm{~K} \cdot \mathrm{min}^{-1}$ during postdeposition cooling. An ambient oxygen pressure of $20 \mathrm{~Pa}$ was used during deposition and cooling.

The room-temperature crystal structure was investigated by high-resolution x-ray diffraction (XRD) on a D8 DISCOVER diffractometer (Bruker corporation) using $\mathrm{Cu} \mathrm{K} \alpha$ radiation. Also $\mathrm{x}$-ray reflectometry studies were performed on this instrument. The diffraction and reflectometry data were fitted using LEPTOS software.

Cross-sectional samples for transmission electron microscopy (TEM) analyses were prepared by focused ion-beam milling in a FEI Quanta 3D instrument. TEM investigations were performed on a FEI Tecnai TF20 X-twin microscope operated at $200 \mathrm{kV}$. The crystal structure was inspected by precession electron diffraction, which minimizes dynamic effects and facilitates automated indexing of the diffraction patterns. Patterns were acquired along different crystallographic directions with a beam $\sim 5 \mathrm{~nm}$ in diameter. Data were collected and processed using ACOM-TEM (Nanomegas, Inc.) software for crystallographic orientation mapping and TOPSPIN (Nanomegas, Inc.) software for strain analysis. The elemental composition of the films was determined by energy dispersive spectroscopy on the same TEM.

For electrical characterization, Pt top electrode pads were formed by pulsed laser deposition using shadow masks. The characterization was carried out on a TF2000 Analyzer (aixACCT Systems GmbH).

\section{B. Modeling}

The results of the phase-field model were obtained by numerical solution of a two-dimensional version of the time-dependent Landau-Ginzburg-Devonshire equation [33], Poisson's equation for dielectrics, and the equation of motion in elastic material, following the models reported in Refs. [34,35]. Elastic, electrostrictive, and certain other material properties were taken from Refs. [32,36-39], while the parameters for the Landau potential were selected to fit the experimental data. The model was numerically solved using the finite element method with a time-dependent solver in COMSOL 5.3. See Supplemental Material B at [40] for more details.

\section{RESULTS}

\section{A. Crystal phases}

The structure of the PSTO/SRO/STO thin films was first analyzed with XRD. Typical XRD $\theta-2 \theta$ scans for the samples are shown in Fig. 1. The scans revealed that the PSTO and SRO films are perovskite, highly oriented, with $(00 l)$ planes parallel to the substrate (001) surface [Fig. 1(a)]. The $(00 l)$ peaks are broad and include peaks from the PSTO and SRO layers. The (001) peak is accompanied by Laue satellites, which are related to the 50-nm-thick SRO layer of high crystal quality [Fig. 1(b)]. A closer inspection of the (004) peak shows a coexistence of different phases or domains in the PSTO films [Fig. 1(c)]. The out-of-plane lattice parameters $c$ for these phases are determined by pseudo-Voigt peak fitting [Figs. 1(d) and $1(\mathrm{e})$ ] and equal to $\sim 3.987,3.978,3.966$, and $3.922 \AA$. Compared with bulk PSTO $(a=b \approx 3.92 \AA, c \approx$ $3.96 \AA$ ), these parameters indicate a complex configuration of structural and ferroelectric domains, which include the outof-plane elongated domains and the out-of-plane contracted ones.

Reciprocal space maps were acquired along different reciprocal lattice points: (003), (303), (204), (224), and (242) (Figs. 2(a)-2(e) and Supplemental Material Fig. S1 [40]). The maps evidence epitaxial relationships for all pseudocubic phases: (001)[100]PSTO \| (001)[100]SRO \| (001)[100]STO. To characterize the phase composition of the PSTO film, the $(a, c)$ lattice parameters were determined from each of the 

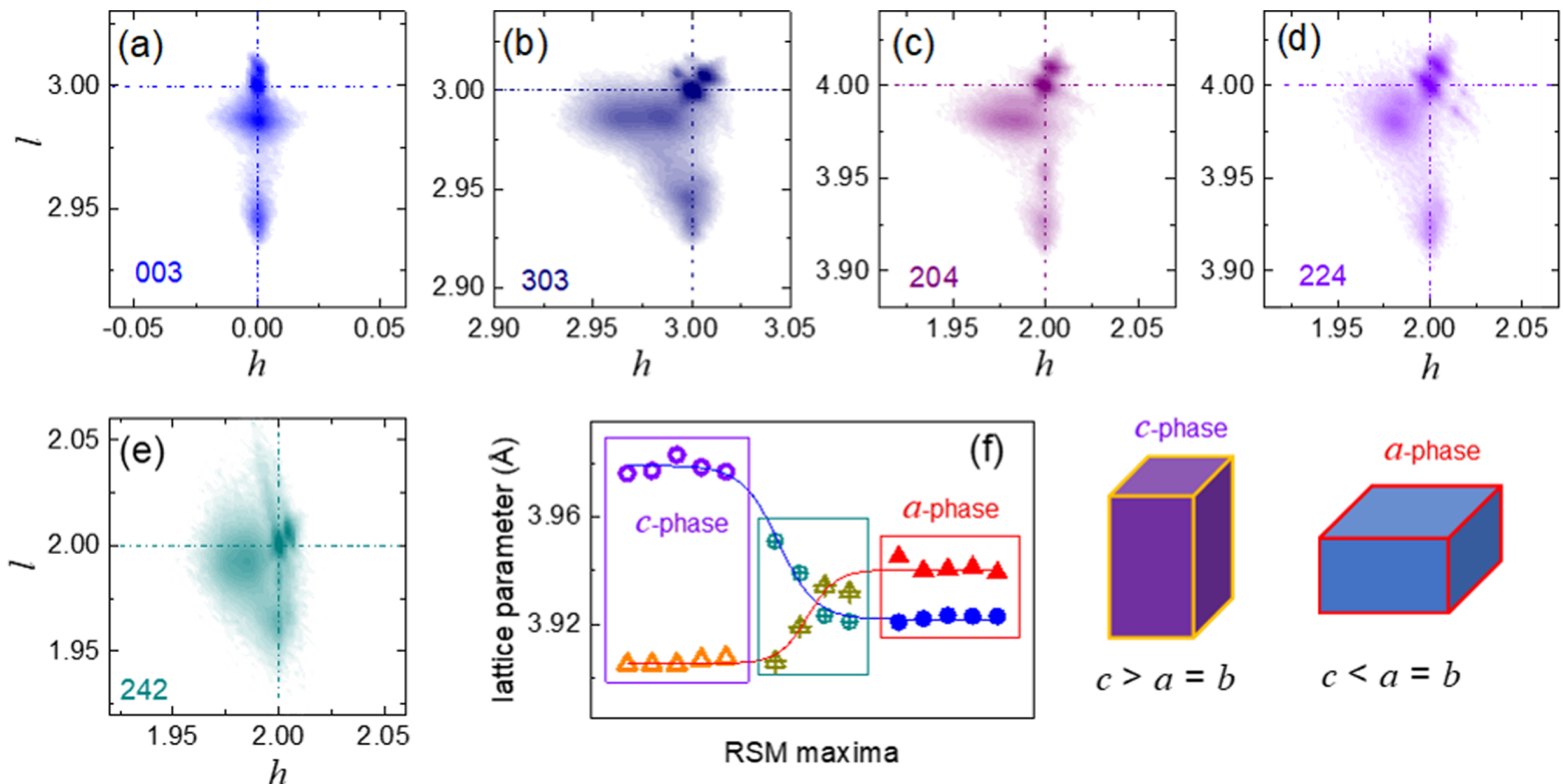

FIG. 2. (a)-(e) X-ray diffraction (XRD) reciprocal space maps along different $h k l$ reciprocal lattice points. Indices are given in reciprocal lattice units of STO. Intensity is shown on logarithmic scale. Positions of the STO peaks are marked by dashed lines. (f) The in-plane (triangles) and out-of-plane (circles) lattice parameters determined from the maps. Schematics of unit cells for the $c$ and $a$ phases are also shown.

intensity maxima in the acquired maps and sorted in order of descending parameter $c$ [Fig. 2(f)]. Although broad and overlapping diffraction peaks make analysis difficult, the acquired data clearly evidence distinct phases. Solid symbols in Fig. 2(f) correspond to a ferroelectric $a$ phase (denoted as $a$-FE here), which is in-plane stretched and out-of-plane compressed: $a=b \approx 3.94 \AA, c \approx 3.92 \AA$. We note that the diffracted intensity is the highest for this phase (see the corresponding peak in Fig. 1). Open symbols are related to a tetragonal $c$ phase ( $c$-FE), which is in-plane compressed, pseudomorphic to STO/SRO, and out-of-plane elongated: $a=b \approx 3.91 \AA, c \approx 3.98 \AA$. The presence of inhomogeneous strain in the $c$-FE phase is manifested by an asymmetric broad (004) $\theta-2 \theta$ peak, which can be formally fitted by two peaks [Fig. 1(d)]. Additionally, the film contains a heterogeneous fraction (crossed symbols), which may also comprise an $r$ phase $(r$-FE).

Although the information on locations of the observed phases is unavailable from the reciprocal space analysis, the results were preliminarily interpreted as follows. The PSTO/STO biaxial in-plane misfit strain is $s_{a}=a_{\mathrm{STO}} / a_{0}-$ $1=0.7 \%$, where $a_{\text {STO }}$ is the lattice parameter of STO, and the lattice parameter $a_{0}$ of unstressed PSTO is extracted from the unit cell volume $V_{0}$ of bulk PSTO: $a_{0}=\left(V_{0}\right)^{1 / 3}$ [32]. This estimation of the misfit strain is valid here because of pseudomorphic epitaxial growth of the SRO bottom electrode layer. The compressive misfit strain explains the formation of the PSTO $c$ phase. The PSTO $a$ phase may originate from a complete relaxation of the misfit strain during high-temperature deposition and buildup of tensile thermal-mismatch strain during postdeposition cooling. Considering the thicknessdependent misfit strain, the $c$ phase can be expected in the vicinity of the SRO-STO interface. Exact spatial locations of the $a$ phase and heterogeneous layer are difficult to determine by XRD.

\section{B. Strain gradient}

To scrutinize the phase composition and investigate spatial phase distribution, we performed a cross-sectional TEM analysis of the elemental composition, crystal phases, and local lattice strains (Figs. 3 and 4 and Supplemental Material Figs. S2-S7 [40]). Whereas significant changes of lattice strain were found across the thickness, such changes were not detected in composition.

Converse to stripelike tilted $a$ domains that are often observed in epitaxial films of tetragonal $\mathrm{PbTiO}_{3}$ and $(\mathrm{Pb}, \mathrm{Zr}) \mathrm{TiO}_{3}$, such domains are absent in the PSTO film. Strain maps were extracted from the diffraction patterns and quantified using the STO substrate as a reference. In

(a)

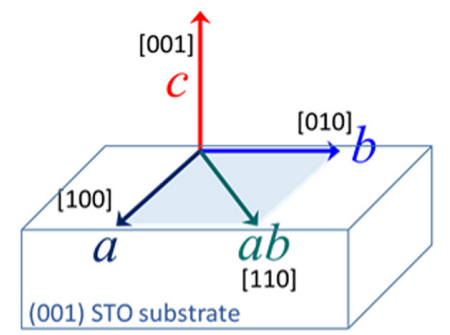

(b)

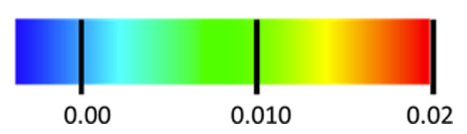

FIG. 3. (a) Schematics of crystallographic directions and (b) color scale for strain mapping. 
(a)

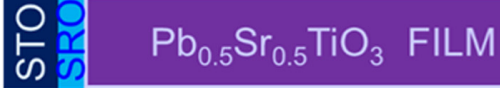

(b)

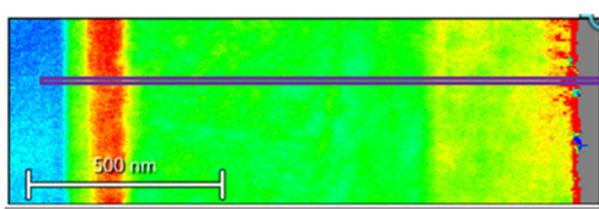

(c)

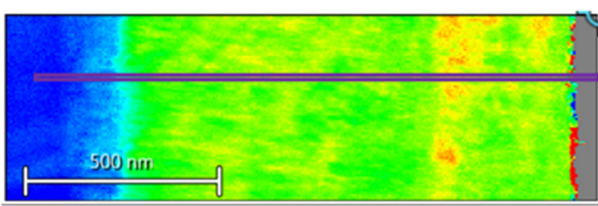

(d)

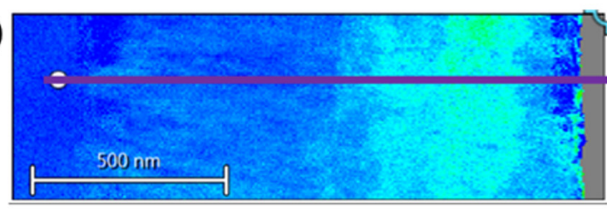

(e)

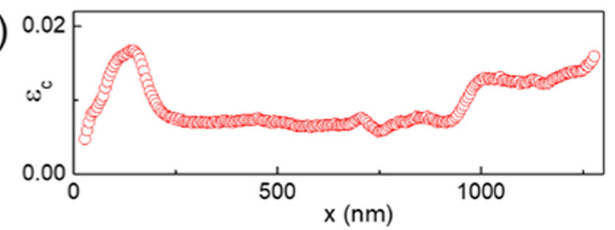

(f)

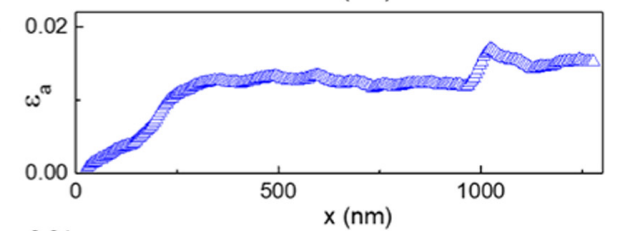

(g)

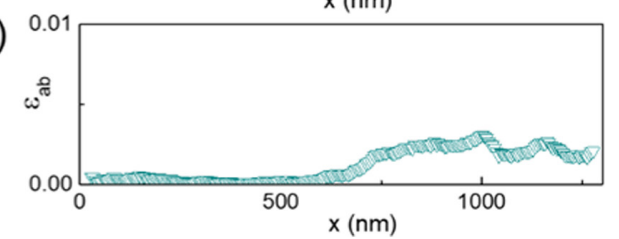

FIG. 4. Cross-sectional (a) schematics of the stack and (b)-(d) strain maps for the $\langle 100\rangle$ crystal direction normal to the substrate surface: along the (b) out-of-plane $c$ direction, (c) in-plane $a$ direction, and (d) in-plane $a b$ direction. Strain as a function of the out-of-plane coordinate along the (e) $c$, (f) $a$, and (g) $a b$ directions. Position of $x=0$ is shown in (d). Strain is quantified with relation to the STO substrate.

Fig. 4(b), the out-of-plane $c$ map contains three distinct regions in the PSTO film: a near-electrode strongly strained layer $\left(c>a_{\mathrm{STO}}\right)$, a central layer $\left(c<a_{\mathrm{STO}}\right)$, and a topmost layer $\left(c \sim a_{\text {STO }}\right)$. Additionally, a strained SRO film is clearly seen in the $c$ map. The in-plane $a$-direction map [Fig. 4(c)] confirms the presence of the three regions in the PSTO film and indicates good in-plane coherence of the SRO film and the bottom layer of the PSTO film to the STO substrate ( $a=$ $a_{\mathrm{STO}}$ ). The in-plane $a b$-direction map [Fig. 4(d)] is uniform for the STO substrate, the SRO film, and a large fraction of the PSTO film. This observation implies that the in-plane lattice parameters are equal $(a=b)$ therein. Interestingly, a marginal difference between the in-plane parameters $(a \neq b)$ is found in the upper layer of the PSTO film [light blue color in Fig. 4(d)].

The presence of different regions seen in the $\langle 100\rangle$ maps (Fig. 4) was confirmed by the maps acquired along the $\langle 110\rangle$ direction (Supplemental Material Figs. S5 and S6 [40]) and by the orientation map (Supplemental Material Fig. S7 [40]).

To relate the measurements in Fig. 4 with the crystal phases and lattice strain in the PSTO film, we quantified the tetragonality $c / a$, the in-plane ratio of the lattice parameters $b / a$, and the in-plane strain $s_{a}=\left(a / a_{0}-1\right)$ as a function of the out-of-plane coordinate in the PSTO film (Fig. 5). The tetragonality $(c / a>1)$ and compressive in-plane strain $\left(s_{a}<0\right)$ are found for $x<200 \mathrm{~nm}$, suggesting the presence of the $c$ phase therein. At larger distances from the substrate, where $200 \mathrm{~nm}<x<950 \mathrm{~nm}$, the tetragonality $(c / a<1)$ and tensile in-plane strain $\left(s_{a}>0\right)$ indicate the presence of the $a$ phase. The in-plane tensile strain $s_{a} \approx 0.5 \%$ is stabilized when $x>$ $350 \mathrm{~nm}$. Importantly, the lattice strain strongly changes with coordinate $x$ for $x<350 \mathrm{~nm}$, showing the presence of a strain gradient. Assuming a linear approximation, the strain gradient is $\sim \geqslant 3 \times 10^{4} \mathrm{~m}^{-1}$. On the top of the film, the detected partial loss of epitaxial coherence (Supplemental Material Fig. S7
[40]) and the measured lattice parameters suggest diverse polarization directions that can be presented as $r$-FE phase.

Thus, a distribution of strain with a distinct strain gradient across the thickness of the film is found in the epitaxial PSTO film on compressive STO substrate. The inhomogeneous strain induces phase transformations as a function of the out-of-plane coordinate. Further, to assess the functionality

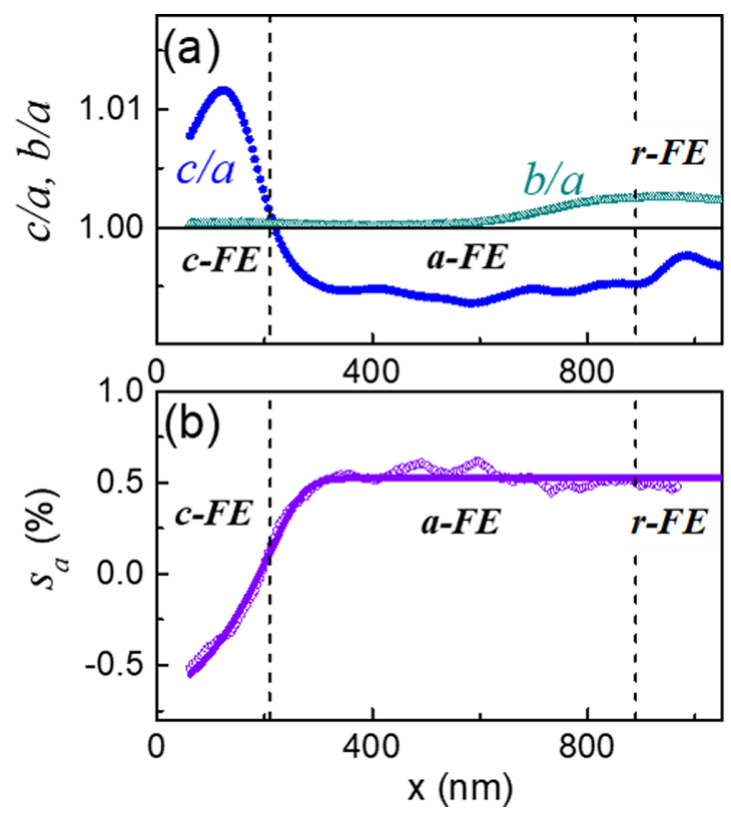

FIG. 5. (a) Ratios of the lattice parameters and (b) in-plane strain as a function of coordinate in the PSTO film. Solid curve shows fit used in the numerical model (b). Vertical dashed lines mark approximate positions of phase boundaries. 

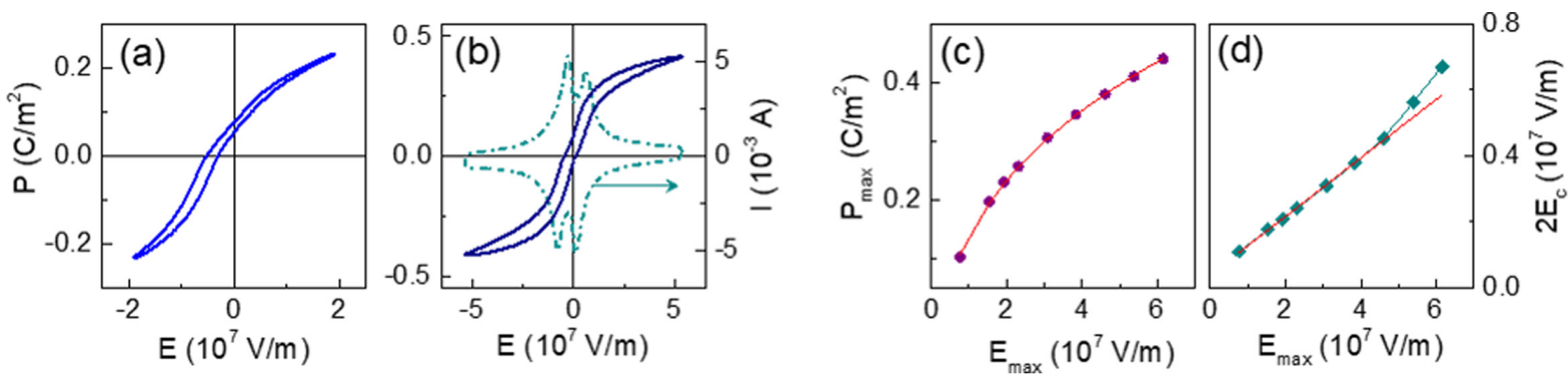

FIG. 6. (a) and (b) Dynamic polarization-electric field and (b) current-electric field loops (dashed curves) acquired at frequency $1 \mathrm{kHz}$ and different maximum applied fields $E_{\max }$. (c) Maximum polarization and (d) width of polarization loops as a function of maximum applied field. Solid red curves show fits to (c) $\left[P_{\max } \propto\left(E_{\max }\right)^{1 / 3}\right]$ and (d) $\left[2 E_{c} \propto E_{\max }\right]$.

and gain more information on ferroelectric phases in the film, we studied polarization response to electric field.

\section{Polarization}

The structural and, hence, ferroelectric phase transformation manifests itself in polarization behavior (Fig. 6). The dynamic $P-E$ loops are slim, and the $I-E$ loops have four peaks, implying two different processes. We note that slim loops are observed at very small fields $\left( \pm E_{\max }\right)$ [Fig. 6(a)]. They contrast with Rayleigh-type loops, which are typical for normal ferroelectrics at subswitching fields, and with the pinched loops, which are caused by defects. The maximum polarization $P_{\max }$ increases with the maximum electric field $E_{\max }$ as $P_{\max } \propto\left(E_{\max }\right)^{1 / 3}$, and the width $2 E_{c}$ of the loops increases as $2 E_{c} \propto E_{\max }$ [Figs. 6(c) and 6(d)]. Such a concurrent scaling of $P_{\max }$ and $2 E_{c}$ is characteristic of field-induced polarization rotation (Supplemental Material Fig. S8 [40]), which was previously found in the $a$ phase of epitaxial $\mathrm{BaTiO}_{3}$ films [41]. The results shown in Figs. 6(c) and 6(d) imply a significant contribution of polarization rotation to the observed switching behavior. A deviation from the linear behavior [ $\left.2 E_{\mathrm{c}} \propto E_{\max }\right]$ at $E_{\max }>5 \times 10^{5} \mathrm{~V} / \mathrm{m}$ [Fig. 6(d)] may indicate an increased contribution of ordinary polarization inversion in the $c$ region. We note that maximum polarization at large fields is $\sim 0.5 \mathrm{C} / \mathrm{m}^{2}$, which is significantly larger than spontaneous polarization of $\sim 0.25 \mathrm{C} / \mathrm{m}^{2}$ in PSTO ceramics and may be related to the strain-enhanced polarization in the near-electrode $c$ phase.

\section{Phase transformations}

To get insight into mechanisms responsible for the observed phase transformations, we performed a theoretical analysis of the strain state and polarization in these films. We used a phase field model following the approach of Ref. [35]. The model incorporated coupling between the ferroelectric and elastic properties of the film. In $\mathrm{Pb}$-containing perovskite oxide ferroelectrics, a unit cell elongates along the polarization direction, as controlled by the electrostrictive coefficients $q_{11}>0$ and $q_{12}<0$. The strain maps and their analysis (Figs. 4 and 5, and Supplemental Material Fig. S5 [40]) revealed three distinct phases, which are distributed across the thickness of the film. The polarization direction is out-ofplane in the near-electrode $c$-FE phase $(c / a>1)$, in-plane in the $a$-FE phase $(c / a<1)$, and has diverse orientations in the $r$-FE phase $(b / a \neq 1)$ that was presented as a sequence of the tetragonal $(c)$, orthorhombiclike $(a)$, and rhombohedrallike or monocliniclike $(r)$ phases in the model. Because of different out-of-plane polarization components in these regions, there is a nonzero net bound charge on the borders between the regions. This bound charge is known to be screened by free charge carriers [42]. Therefore, corresponding charged layers were introduced in the model. The possible mechanisms for the appearance and transport of the free carriers are discussed in Ref. [42] and references therein. Apart from this feature, the film was described in the dielectric approximation. The electrostrictive and elastic coefficients were taken from the known properties of PTO. The Landau coefficients of PSTO are not tabulated. In view of the expected proximity between the paraelectric and ferroelectric phases [32], a thermodynamic potential of the type $\Phi=\frac{\gamma}{6} P^{2}\left(P^{2}-P_{s}^{2}\right)^{2}$ was used as a benchmark, with the spontaneous polarization $P_{s}=0.3 \mathrm{C} / \mathrm{m}^{2}$ as in PSTO ceramics. Anisotropic corrections to the potential were established from the best fits to the experimental data. Additionally, the experimentally observed strain inhomogeneities were approximated using random charged defects with a linear bottom-to-top increase of the strength of random fields. More details of the simulations are given in Supplemental Material B [40].

To analyze the strain distribution (Fig. 7), the average in-plane strain $u_{x x}$ was set using the Dirichlet boundary conditions for displacement on the lateral sides and considering the results shown in Fig. 5(b). The ferroelectric properties of the system were then uncovered by fitting the out-of-plane strain profile and the hysteresis loops. The calculated out-of-plane strain $u_{z z}$ agreed with the measurements when we introduced the $c-a$ and $a-r$ interphase charged layers with an absolute integral charge density of $0.28 \mathrm{C} / \mathrm{m}^{2}$ in each layer. The same and opposite signs of the charges of interphase layers were considered. Hereafter, we present the results for the charged layers of the same sign (see Supplemental Material C [40] for the other case). Notably, matching between the in-plane and out-of-plane strains was impossible to achieve without introducing interphase charges. An alternative interpretation would require severe compositional changes across the thickness of the film, at the level of a fourfold change in the $\mathrm{Pb}: \mathrm{Sr}$ ratio. Such hypothetical changes are in strong conflict with the compositional uniformity, which is common for pulsed laser deposition and confirmed here. 
(a)

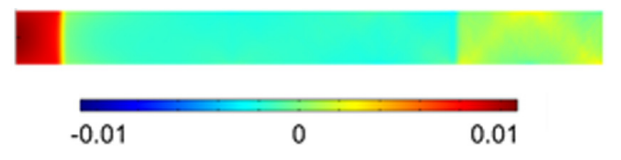

(b)

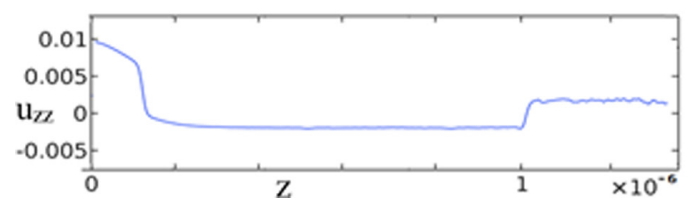

(c)

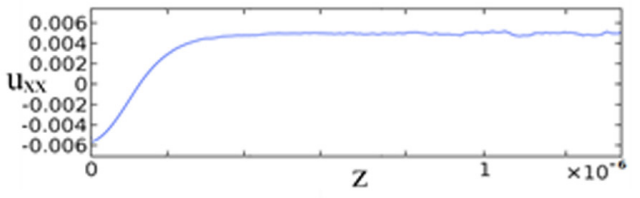

FIG. 7. (a) and (b) Calculated strain distribution: (a) color map and (b) distribution along the central line for the out-of-plane strain $u_{z z}$ and (c) distribution along the central line for the in-plane strain $u_{x x}$.

Thus, the observed phase transformations inside the film result from the synergetic relaxation of misfit strain and charge screening. Whereas strain relaxation and straindependent crystal phases are commonly accepted for epitaxial ferroelectric films, the revealed presence of interphase charges is seldom considered. Interestingly, the interphase charges are built of charge carriers, which are trapped at certain locations in the film. Considering the electronic band structure, this trapping suggests occupied in-gap states (i.e., states in the energy gap between the conduction and valence bands). Because electronic excitations/relaxations from/to these states can significantly contribute to charge transport [43,44], strain-induced structural features may control electrical conductivity. Such control is of special interest for resistive switching, important for advanced ferroelectric diodes and memristors.

Further on, we calculated the polarization and the polarization-electric field hysteresis loops (Fig. 8). The outof-plane polarization, or $z$ component, is nearly homogeneous in each of the regions because it is governed by the minimization of the electrostatic energy. The in-plane polarization component tends to take either value of the two minima with equal probability. This behavior leads to the presence of ferroelastic domain walls. These tendencies are expected, although they can be more complicated, also in a real threedimensional film. The simulated polarization-field hysteresis loop [Fig. 8(d)] qualitatively agrees with the experimental observations [Fig. 6(a)]: both the measured and calculated loops are slim and shifted along the field axis. We note that the electric field can stimulate electronic excitations, imposing changes in the interphase charges that are not accounted for in

(a)

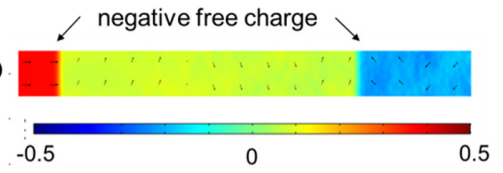

(b)

(c)
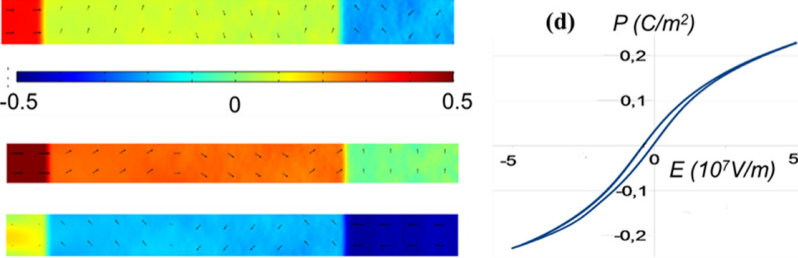

FIG. 8. Simulated polarization (a) in a short-circuited sample and (b) and (c) under application of electric field of $\pm 10^{7} \mathrm{~V} / \mathrm{m}$. Arrows show polarization vectors; color shows out-of-plane $z$ component. (d) Simulated hysteresis loop. the model. Such excitations are nearly unstudied, and their theoretical description presents a challenge for subsequent research.

\section{DISCUSSION}

The observed structural changes involve polarization change and strain gradient. In this context, a discussion of the role of the flexoelectricity is natural. We note that the observed effects of strain gradient on polarization state in the film are not due to the flexoelectric effect as such. The classical theory of flexoelectricity would predict $\Delta P_{i}=\chi_{m i} f_{k l m j} \partial u_{k l} / \partial x_{j}$, where $f_{k l m j}$ of the order of Volts are flexocoupling coefficients, and $\chi_{m i}$ is the dielectric susceptibility. Substituting the above results - a characteristic change of strain at $1 \%$ over $100 \mathrm{~nm}$-we get $\Delta P \sim 0.001-0.01 \mathrm{C} / \mathrm{m}^{2}$. Here, we assume the relative susceptibility in PSTO is of the order of 100-1000. Thus, the observed polarization change is one to two orders of magnitude higher than one would expect from the classical bulk flexoelectric effect. Interestingly, a possible interpretation of the observed phenomena in terms of flexoelectricity would yield effectively much larger flexocoupling constants, of the order of 100-1000 V. Such apparent gigantic constants were previously reported in bulk samples, whose phase composition was not inspected [45]. The results of this paper imply that apparent giant flexoelectricity can be caused by phase transitions in resemblance with enhanced piezoelectric response in morphotropic phase boundary materials. Proximity to a phase transition makes polarization susceptible to both strain and strain gradient.

It is worth mentioning that the relaxation of strain and, hence, generation of strain gradients are common for many heteroepitaxial films that point to a general possibility for gradient-induced ferroelectric phases (Supplemental Material D [40]). We also note that ferroelectric phases and, correspondingly, complex phase composition in such films are formed on cooling after high-temperature deposition. As known from strain-temperature phase diagrams of epitaxial ferroelectric films [46], the phase-transition temperature $T_{\mathrm{PT}}$ increases linearly with magnitude of in-plane strain therein. Therefore, the presence of coordinate-dependent in-plane strain determines a coordinate-dependent temperature $T_{\mathrm{PT}}$. In the studied PSTO film, the temperature $T_{\mathrm{PT}}$ is highest in the near-substrate region, suggesting that, on cooling, the tetragonal ferroelectric $c$ fraction is formed first, which is followed by appearance of spontaneous polarization at lower $T_{\mathrm{PT}}$ in 
the rest of the film. Because polarization states in the layers are coupled, phase transitions are expected to occur by steps and synchronously with the compensation of the corresponding charged boundaries which are relatively energetically expensive $\left(\sim \mathrm{J} / \mathrm{m}^{2}\right)$ [41]. However, to get an explicit picture, accurate cross-sectional measurements of strain should be carried out as a function of high temperatures, which represents a separate challenging task.

\section{CONCLUSIONS}

Epitaxial stacks of perovskite oxide ferroelectric PSTO films and bottom SRO layers were grown on (001) STO substrates. Crystal structure, phases, lattice strains, and polarization were investigated experimentally and theoretically. The distinct phases and distribution of these phases across the thickness of the film as well as the distribution of lattice strains were detected and modeled. It was established that structural changes result from strain gradient accompanied by trapping of charge carriers at the interphase boundaries.
Polarization-electric-field behavior was suggested to involve the rotation and inversion of polarization and the interphase electronic excitations. Like giant piezoelectric response in materials with a morphotropic phase boundary, the observed structural changes may conceptually be interpreted as a giant flexoelectric response to the growth-induced strain gradient due to phase transformations.

\section{ACKNOWLEDGMENTS}

This paper was supported in part by the Operational Program Research, Development and Education, financed by the European Structural and Investment Funds and the Czech Ministry of Education, Youth, and Sports (Project No. SOLID21, CZ.02.1.01/0.0/0.0/16_019/0000760), and the Czech Science Foundation (Grants No. 19-09671S and No. 21-09685S). We acknowledge CzechNanoLab Research Infrastructure supported by MEYS CR (LM2018110). P.Y. acknowledges the Russian Foundation for Basic Research, Grant No. 19-02-00938, simulation of hysteresis loops was carried out under state contract with IT SB RAS (121031200084-2).
[1] G. Catalan, J. Seidel, R. Ramesh, and J. F. Scott, Domain wall nanoelectronics, Rev. Mod. Phys. 84, 119 (2012).

[2] L. McGilly, P. Yudin, L. Feigl, A. Tagantsev, and N. Setter, Controlling domain wall motion in ferroelectric thin films, Nat. Nanotechnol. 10, 145 (2015).

[3] P. Yudin, M. Y. Hrebtov, A. Dejneka, and L. McGilly, Modeling the Motion of Ferroelectric Domain Walls with the Classical Stefan Problem, Phys. Rev. Appl. 13, 014006 (2020).

[4] F. Bruno, K. Rushchanskii, S. Valencia, Y. Dumont, C. Carrétéro, E. Jacquet, R. Abrudan, S. Blügel, M. Ležaić, M. Bibes, and A. Barthélémy, Rationalizing strain engineering effects in rare-earth nickelates, Phys. Rev. B 88, 195108 (2013).

[5] K. J. Choi, M. Biegalski, Y. Li, A. Sharan, J. Schubert, R. Uecker, P. Reiche, Y. Chen, X. Pan, V. Gopalan, L.-Q. Chen, D. G. Schlom, and C. B. Eom, Enhancement of ferroelectricity in strained batio3 thin films, Science 306, 1005 (2004).

[6] L. Chen, Y. Yang, Z. Gui, D. Sando, M. Bibes, X. Meng, and L. Bellaiche, Large Elasto-Optic Effect in Epitaxial $\mathrm{PbTiO}_{3}$ Films, Phys. Rev. Lett. 115, 267602 (2015).

[7] M. Grisolia, F. Bruno, D. Sando, H. Zhao, E. Jacquet, X. Chen, L. Bellaiche, A. Barthélémy, and M. Bibes, Structural, magnetic, and electronic properties of $\mathrm{GdTiO}_{3}$ mott insulator thin films grown by pulsed laser deposition, Appl. Phys. Lett. 105, 172402 (2014).

[8] J. Haeni, P. Irvin, W. Chang, R. Uecker, P. Reiche, Y. Li, S. Choudhury, W. Tian, M. Hawley, B. Craigo, A. K. Tagantsev, X. Q. Pan, S. K. Streiffer, L. Q. Chen, S. W. Kirchoefer, J. Levy, and D. G. Schlom, Room-temperature ferroelectricity in strained $\mathrm{SrTiO}_{3}$, Nature (London) 430, 758 (2004).

[9] R. Zeches et al., A strain-driven morphotropic phase boundary in $\mathrm{BiFeO}_{3}$, Science 326, 977 (2009).

[10] D. Butkovičová, X. Marti, V. Saidl, E. SchmoranzerováRozkotová, P. Wadley, V. Holý, and P. Němec, Critical role of the sample preparation in experiments using piezoelectric actuators inducing uniaxial or biaxial strains, Rev. Sci. Instrum. 84, 103902 (2013).

[11] S. Kondo, T. Yamada, A. K. Tagantsev, P. Ma, J. Leuthold, P. Martelli, P. Boffi, M. Martinelli, M. Yoshino, and T. Nagasaki, Large impact of strain on the electro-optic effect in ( $\mathrm{Ba}, \mathrm{Sr}) \mathrm{TiO}_{3}$ thin films: Experiment and theoretical comparison, Appl. Phys. Lett. 115, 092901 (2019).

[12] D. Thuau, M. Abbas, G. Wantz, L. Hirsch, I. Dufour, and C. Ayela, Mechanical strain induced changes in electrical characteristics of flexible, non-volatile ferroelectric OFET based memory, Org. Electron. 40, 30 (2017).

[13] P. Chidambaram, C. Bowen, S. Chakravarthi, C. Machala, and R. Wise, Fundamentals of silicon material properties for successful exploitation of strain engineering in modern CMOS manufacturing, IEEE Trans. Electron Devices 53, 944 (2006).

[14] D. G. Schlom, L.-Q. Chen, C. J. Fennie, V. Gopalan, D. A. Muller, X. Pan, R. Ramesh, and R. Uecker, Elastic strain engineering of ferroic oxides, MRS Bull. 39, 118 (2014).

[15] A. R. Damodaran, J. C. Agar, S. Pandya, Z. Chen, L. Dedon, R. Xu, B. Apgar, S. Saremi, and L. W. Martin, New modalities of strain-control of ferroelectric thin films, J. Phys. Condens. Matter 28, 263001 (2016).

[16] Y. Feng, Y. Tang, Y. Zhu, M. Zou, and X. Ma, Misfit strain relaxations of (101)-oriented ferroelectric $\mathrm{PbTiO}_{3} /(\mathrm{La}, \mathrm{Sr})(\mathrm{Al}, \mathrm{Ta}) \mathrm{O}_{3}$ thin film systems, J. Mater. Res. 33, 4156 (2018).

[17] J. Speck, A. Daykin, A. Seifert, A. Romanov, and W. Pompe, Domain configurations due to multiple misfit relaxation mechanisms in epitaxial ferroelectric thin films. III. Interfacial defects and domain misorientations, J. Appl. Phys. 78, 1696 (1995).

[18] S. Jain, A. Harker, and R. Cowley, Misfit strain and misfit dislocations in lattice mismatched epitaxial layers and other systems, Philos. Mag. A 75, 1461 (1997). 
[19] J. Speck and W. Pompe, Domain configurations due to multiple misfit relaxation mechanisms in epitaxial ferroelectric thin films. I. Theory, J. Appl. Phys. 76, 466 (1994).

[20] M.-W. Chu, I. Szafraniak, R. Scholz, C. Harnagea, D. Hesse, M. Alexe, and U. Gosele, Impact of misfit dislocations on the polarization instability of epitaxial nanostructured ferroelectric perovskites, Nat. Mater. 3, 87 (2004).

[21] V. Nagarajan, C. Jia, H. Kohlstedt, R. Waser, I. Misirlioglu, S. Alpay, and R. Ramesh, Misfit dislocations in nanoscale ferroelectric heterostructures, Appl. Phys. Lett. 86, 192910 (2005).

[22] C. Jia, S. Mi, K. Urban, I. Vrejoiu, M. Alexe, and D. Hesse, Effect of a Single Dislocation in a Heterostructure Layer on the Local Polarization of a Ferroelectric Layer, Phys. Rev. Lett. 102, 117601 (2009).

[23] P. Gao, C. T. Nelson, J. R. Jokisaari, S.-H. Baek, C. W. Bark, Y. Zhang, E. Wang, D. G. Schlom, C.-B. Eom, and X. Pan, Revealing the role of defects in ferroelectric switching with atomic resolution, Nat. Commun. 2, 591 (2011).

[24] H. Wu, J. Wang, S. Cao, and T. Zhang, Effect of dislocation walls on the polarization switching of a ferroelectric single crystal, Appl. Phys. Lett. 102, 232904 (2013).

[25] Y. Tang, Y. Zhu, Y. Liu, Y. Wang, and X. Ma, Giant linear strain gradient with extremely low elastic energy in a perovskite nanostructure array, Nat. Commun. 8, 15994 (2017).

[26] P. Yudin and A. Tagantsev, Fundamentals of flexoelectricity in solids, Nanotechnology 24, 432001 (2013).

[27] A. K. Tagantsev and P. V. Yudin, Flexoelectricity in Solids (World Scientific, Singapore, 2016).

[28] D. Sando, M. Han, V. Govinden, O. Paull, F. Appert, C. Carrétéro, J. Fischer, A. Barthélémy, M. Bibes, V. Garcia, S. Fusil, B. Dkhil, J. Juraszek, Y. Zhu, X. Ma, and V. Nagarajan, Interfacial strain gradients control nanoscale domain morphology in epitaxial $\mathrm{BiFeO}_{3}$ multiferroic films, Adv. Funct. Mater. 30, 2000343 (2020).

[29] K.-E. Kim, S. Jeong, K. Chu, J. H. Lee, G.-Y. Kim, F. Xue, T. Y. Koo, L.-Q. Chen, S.-Y. Choi, R. Ramesh, and C.-H. Yang, Configurable topological textures in strain graded ferroelectric nanoplates, Nat. Commun. 9, 403 (2018).

[30] Y. L. Tang, Y. L. Zhu, X. L. Ma, A. Y. Borisevich, A. N. Morozovska, E. A. Eliseev, W. Y. Wang, Y. J. Wang, Y. B. Xu, Z. D. Zhang, and S. J. Pennycook, Observation of a periodic array of flux-closure quadrants in strained ferroelectric $\mathrm{PbTiO}_{3}$ films, Science 348, 547 (2015).

[31] A. Yadav, C. Nelson, S. Hsu, Z. Hong, J. Clarkson, C. Schleputz, A. Damodaran, P. Shafer, E. Arenholz, L. Dedon, D. Chen, A. Vishwanath, A. M. Minor, L. Q. Chen, J. F. Scott, L. W. Martin, and R. Ramesh, Observation of po- lar vortices in oxide superlattices, Nature (London) 530, 198 (2016).

[32] H. Landolt and R. Bornstein, Numerical Data and Functional Relationships in Science and Technology: Astronomy, Astrophysics and Space Research, New Series, Group 6 (Springer, Berlin, 1965), Vol. 1.

[33] S. Semenovskaya and A. Khachaturyan, Development of ferroelectric mixed states in a random field of static defects, J. Appl. Phys. 83, 5125 (1998).

[34] Y. Li and L. Chen, Temperature-strain phase diagram for $\mathrm{BaTiO}_{3}$ thin films, Appl. Phys. Lett. 88, 072905 (2006).

[35] T. Sluka, K. G. Webber, E. Colla, and D. Damjanovic, Phase field simulations of ferroelastic toughening: the influence of phase boundaries and domain structures, Acta Mater. 60, 5172 (2012).

[36] M. Haun, Z. Zhuang, E. Furman, S. Jang, and L. E. Cross, Thermodynamic theory of the lead zirconate-titanate solid solution system, part III: Curie constant and sixth-order polarization interaction dielectric stiffness coefficients, Ferroelectrics 99, 45 (1989).

[37] N. Pertsev, V. Kukhar, H. Kohlstedt, and R. Waser, Phase diagrams and physical properties of single-domain epitaxial $\mathrm{Pb}\left(\mathrm{Zr}_{1-x} \mathrm{Ti}_{x}\right) \mathrm{O}_{3}$ thin films, Phys. Rev. B 67, 054107 (2003).

[38] J. Hlinka, P. Ondrejkovic, and P. Marton, The piezoelectric response of nanotwinned $\mathrm{BaTiO}_{3}$, Nanotechnology 20, 105709 (2009).

[39] A. K. Tagantsev, Landau expansion for ferroelectrics: which variable to use? Ferroelectrics 375, 19 (2008).

[40] See Supplemental Material at http://link.aps.org/supplemental/ 10.1103/PhysRevResearch.3.033213 for: (A) Supplementary experimental figures; (B) The phase field model; (C) The results for oppositely charged layers; (D) Universal behavior.

[41] M. Tyunina, O. Pacherova, J. Perantie, M. Savinov, M. Jelinek, H. Jantunen, and A. Dejneka, Perovskite ferroelectric tuned by thermal strain, Sci. Rep. 9, 3677 (2019).

[42] P. S. Bednyakov, B. I. Sturman, T. Sluka, A. K. Tagantsev, and P. V. Yudin, Physics and applications of charged domain walls, npj Computational Materials 4, 65 (2018).

[43] M. Tyunina and M. Savinov, Charge transport in epitaxial barium titanate films, Phys. Rev. B 101, 094106 (2020).

[44] M. Tyunina, Conductivity in ferroelectric barium titanate: electrons versus oxygen vacancies, IEEE Trans. Ultrason. Ferroelectr. Freq. Control 68, 296 (2020).

[45] W. Ma and L. E. Cross, Flexoelectric polarization of barium strontium titanate in the paraelectric state, Appl. Phys. Lett. 81, 3440 (2002).

[46] N. Pertsev, A. Zembilgotov, and A. Tagantsev, Effect of Mechanical Boundary Conditions on Phase Diagrams of Epitaxial Ferroelectric Thin Films, Phys. Rev. Lett. 80, 1988 (1998). 